\title{
Directional uv photoemission from (100) and (110) molybdenum surfaces
}

Cinti, R. C.; Khoury, E. Al; Chakraverty, B. K.; Christensen, N. E.

Published in:

Physical Review B

Link to article, DOI:

10.1103/PhysRevB.14.3296

Publication date:

1976

Document Version

Publisher's PDF, also known as Version of record

Link back to DTU Orbit

Citation (APA):

Cinti, R. C., Khoury, E. A., Chakraverty, B. K., \& Christensen, N. E. (1976). Directional uv photoemission from (100) and (110) molybdenum surfaces. Physical Review B, 14(8), 3296-3300.

https://doi.org/10.1103/PhysRevB.14.3296

\section{General rights}

Copyright and moral rights for the publications made accessible in the public portal are retained by the authors and/or other copyright owners and it is a condition of accessing publications that users recognise and abide by the legal requirements associated with these rights.

- Users may download and print one copy of any publication from the public portal for the purpose of private study or research.

- You may not further distribute the material or use it for any profit-making activity or commercial gain

- You may freely distribute the URL identifying the publication in the public portal 


\title{
Directional uv photoemission from (100) and (110) molybdenum surfaces
}

\author{
R. C. Cinti, E. Al Khoury, and B. K. Chakraverty \\ Groupe des Transitions de Phases, Centre National de la Recherche Scientifique, Boite Postale 166X, 38042 Grenoble, France
}

N. E. Christensen

Physics Laboratory I, The Technical University of Denmark, D.K. 2800 Lyngby, Denmark

(Received 1 December 1975)

\begin{abstract}
A study of the (100) and (110) molybdenum surfaces by directional photoemission spectroscopy is presented. Energy distribution spectra formed by photoelectrons emitted normal to the surfaces have been measured for photon energies between 10.2 and $21.2 \mathrm{eV}$. The results are discussed in terms of calculated band structure within the framework of the $K_{\|}$-conservation assumption. A good agreement is found between the main features of the experimental spectra and the emission expected from the band structure along the corresponding symmetry line in the Brillouin zone, assuming essentially direct-transition and surface-emission processes. Two extra structures are interpreted in terms of surface states or resonances: on the (100) surface, $0.5 \mathrm{eV}$ below $E_{F}$; on the (110) surface, $4.5 \mathrm{eV}$ below $E_{F}$ in the $s-d$ hybridization gap.
\end{abstract}

\section{INTRODUCTION}

In the past few years there has been considerable interest centered on photoemission spectroscopy in obtaining experimental information on the electronic structures of metals, ${ }^{1}$ both bulk and surface contributions. Excellent results were obtained specially on noble metal $\mathrm{s}^{2,3}$ where good agreement was found between experimental spectra and calculated energy distributions of the joint densities of states. In more recent work, ${ }^{4}$ the directional properties of photoemission were explored. Assuming free-electron refraction conditions for photoelectrons escaping through the surface, encouraging results were obtained in checking calculated energy-vs-wave-vector dispersion relations for electronic states along simple directions of the Brillouin zone.

A major difficulty encountered in this kind of study is to define a coherent theoretical framework to connect experimental spectra to the calculated band structures of the solids studied. The semiphenomenological three-step model of Berglund and Spicer ${ }^{5}$ has given a good description of a number of experimental results but shows some failures in the case of transition metals. ${ }^{4}$ Moreover, this model has been criticized in more rigorous analyses ${ }^{6-8}$ of the photoemission process which are, however, as yet difficult to handle for realistic cases; having been carried out only for simple-metal models.

Directional photoemission spectra of the (100) and (110) faces of molybdenum will be presented, and these experimental results are compared to a relativistic augmented-plane-wave (RAPW) bandstructure calculation. This interpretation follows the lines used for tungsten, ${ }^{4}$ for which it appears possible to separate "bulk emission" from "surface emission" processes or "band-gap emission" as discussed by Eastman and Feibelman. ${ }^{9}$ We have not tried to compare our results with other measurements on molybdenum obtained under rather different experimental conditions. ${ }^{10-12}$ Conversely we will compare them to results obtained by the same experimental method on tungsten $^{4}$ which has a band structure that is similar to that of molybdenum.

\section{EXPERIMENT}

Photoelectron spectra were measured using a cylindrical mirror analyzer having a theoretical resolution of $1 \%$. This experimental arrangement was partially obstructed to count only electrons emitted within a cone of $10^{\circ}$ full opening centered about the normal to the sample. Transmitted electrons were detected by a channeltron electron multiplier and the counts were stored in a 400 channel multiscaler whose channel address was controlled directly by the plate potential of the analyzer.

The photon source was a low-pressure Damany discharge lamp"13 associated with a "CreusotLoire" monochromator of the Pouey type, ${ }^{13}$ giving six photon energies: $10.2,11.8,13.5,16.8,18.4$, and $21.2 \mathrm{eV}$. The beam impinged the crystal at an incidence angle of about $50^{\circ}$ after a reflection on a platinized toroidal mirror focusing the radiation on the sample. Differential pumping along this line and a large capacity pumping system for the working chamber kept the residual pressure at about $10^{-9}$ Torr during measurements.

A mass spectrometer and an Auger analysis system within the experimental chamber allowed us 
to check the composition of the residual atmosphere and the crystal contamination. The single crystals used in this experiment were cut along the respective crystallographic planes to within $2^{\circ}$. They had a ribbon shape allowing resistive heating. After good mechanical polishing and electropolishing they were cleaned in situ by conventional thermal and oxygen treatments and checked by Auger spectroscopy which revealed no presence of carbon or oxygen. A clean surface was then restored whenever necessary by flash heating to $2000^{\circ} \mathrm{C}$.

In the pressure conditions used we found that a time of $3 \mathrm{~min}$. elapsed before the beginning of contamination which was detectable by Auger analysis or by some modification of the spectra themselves. For this reason we have worked with scan times of $60 \mathrm{sec}$ between successive heat pulses which clean the crystal and ensure surface purity. In the spectra presented here, no correction for the variation of analyzer resolution and transmission with energy was applied. The bottom scale refers to the initial-state energy of the electrons before excitation relative to the Fermi level. The origin of this scale is positioned within an accuracy of $0.2 \mathrm{eV}$ owing to the difficulty of knowing the analyzer work function precisely.

\section{RESULTS}

\section{A. Emission normal to the (100) face}

Figure 1 shows a set of photoelectron energy distribution curves obtained for excitation energies between 10.2 and $21.2 \mathrm{eV}$, their relative intensity being arbitrarily normalized for presentation. The left-hand side gives the corresponding section of the band structure as obtained by the relativistic augmented-plane-wave method. These spectra show two different kind of structures. (a) Two peaks, labeled $B$ and $E$, varying in their initialstate energy with $\hbar \omega$ in the way characteristic for emission due to optical direct transition. (b) Three other peaks, marked $A, C$, and $D$, originate from constant initial-state levels situated, respectively, at $-0.5,-1.7$, and $-3.5 \mathrm{eV}$ below the Fermi level. Structure $E$ is unquestionably due to a direct transition between the two first bands nearly degenerate near the point $H$ and the empty band 6 . The agreement between the expected and the observed structure is very good here. In the same way, peak $B$ can be attributed to a direct transition between the quasiflat part of bands 2 and 3 near point $\Gamma$ and the parabolic part of empty bands 7-9. Here the agreement is poorer than in the previous case. A better fit would occur if a slight correction could be made on the calculated band struc- ture as is shown by a dashed line in the band diagram of Fig. 1.

The stationary peaks $C$ and $D$, reflecting initial densities of states, can be interpreted in terms of nondirect transition process or by surface emission. The similarity of our results with the comparable data obtained for tungsten ${ }^{4}$ (we will discuss this below), where the nondirect process is found to be unimportant, supports the second hypothesis. Peak $C$ is therefore correlated to the high density of states associated with the minima (modified) in bands 2 and 3 near point $\Gamma$, and the prominent feature, $D$, corresponds to the maximum in the lowest band on the middle of the $\Delta$ line. To fit the energies correctly it is necessary to shift this maximum to higher values by about $0.5 \mathrm{eV}$.

The last structure, $A$, can be directly compared to a similar feature observed previously on tungsten by photoemission ${ }^{4,14,15}$ and field emission ${ }^{16}$ spectroscopy and assigned to emission from a surface resonant level. Theoretically this extra

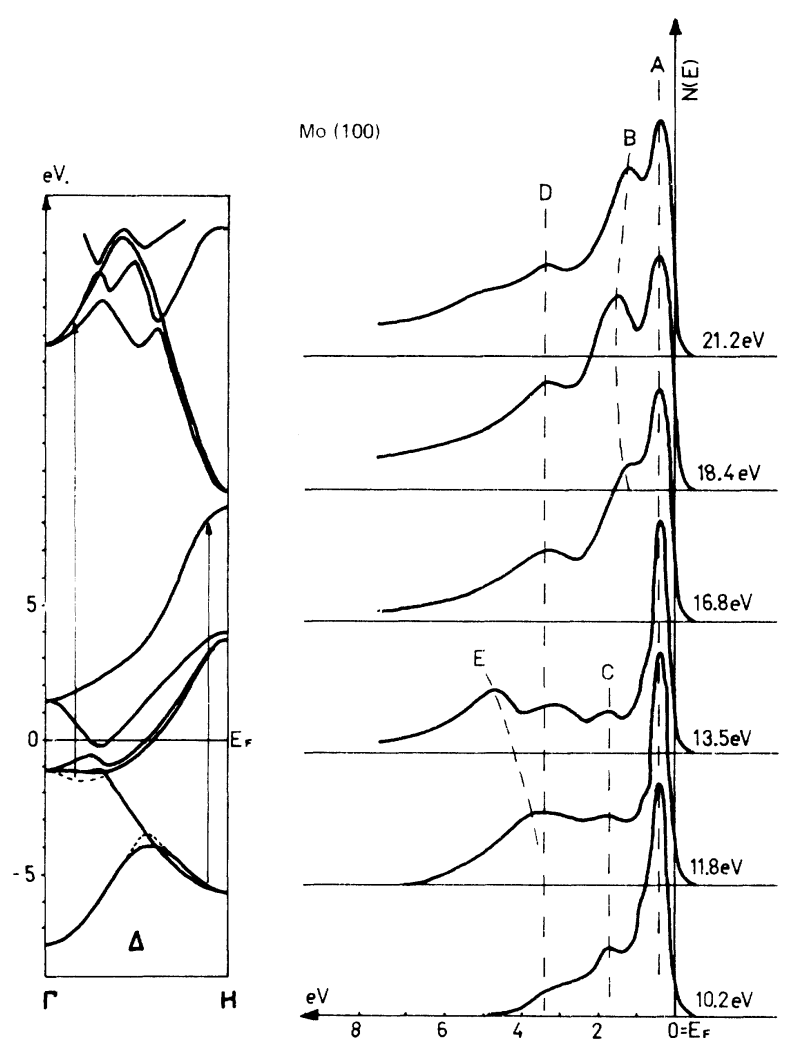

FIG. 1. Experimental spectra of photoelectrons emitted normal to the $(100)$ surface for photon energies between 10.2 and $21.2 \mathrm{eV}$ (right-hand side). Calculated band structure of molybdenum along the $\Gamma H$ symmetry line where the observed direct transitions are indicated by arrows (left-hand side). 
density of states has been predicted by CyrotLackmann and Desjonquères ${ }^{17}$ near the middle of the $d$ band in bcc transition metals and also by Sturm ${ }^{18}$ in the band gap caused by the spin-orbit coupling just below the Fermi level along the $\Gamma H$ direction in the same materials. As previously observed in tungsten, this structure is very sensitive to surface contamination by adsorbed gases, this fact giving good evidence for its surface origin. Figure 2 shows the evolution of a spectrum with the exposure time to a residual pressure in the working chamber of $10^{-9}$ Torr composed of $50 \% \mathrm{Ne}, 20 \% \mathrm{CO}$, and $10 \% \mathrm{CO}_{2}$, the rest being $\mathrm{H}_{2}$ and $\mathrm{CH}_{4}$ in equal parts. The intensity of the first peak decreases quickly with time, while the other structures are more stable and a contamination peak due to $\mathrm{CO}$ grows at $-6 \mathrm{eV}$ below $E_{F}$. However, in spite of its large sensitivity it does not vanish completely and this behavior suggests a double origin for this peak, partially due both to the resonance states and to a surface emission associated with the maxima of density corresponding to the extrema of bands 4 and 5 around the spin-orbit gap. We have not observed emission from high densities due to the flat parts of the lowest band near the symmetry points $\Gamma$ and $H$.

\section{B. Emission normal to the (110) face}

Figure 3 shows results obtained from the (110) surface for the six photon energies used with the corresponding calculated band-structure section along the $\Gamma N$ symmetry line. As for the (100) results, the relative intensity of the spectra are arbitrarily normalized for presentation. The structures can be interpreted as follows.

(a) Peak $D$, varying in initial energy with $\hbar \omega$, may be assigned to photoemission following direct

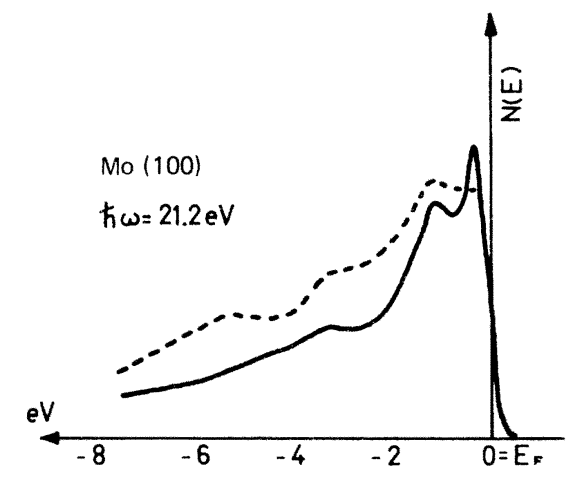

FIG. 2. Effect of surface contamination on a spectrum measured on the (100) face at $21.2-\mathrm{eV}$ photon energy. Full-line curve, clean surface; dashed-line curve, surface exposed $15 \mathrm{~min}$ to the residual atmosphere of $10^{-9}$ Torr. transitions between the second band and the parabolic parts of bands 7 and 8 near point $N$. In fact, to have a better fit here it would be necessary to correct the band scheme in the manner shown on the figure by dashed lines. We see that if the correction applied on the filled band is slight, the shift applied on the empty state band is drastic and decreases the large band gap by about $3 \mathrm{eV}$ near point $N$.

(b) Peaks $A, B$, and $C$, stable in initial energy as indicated by the vertical lines, located, respectively, at $0.5,1.0$, and $1.6 \mathrm{eV}$ below the Fermi level are found to originate from constant initial states. The last two, $B$ and $C$, reflect by a surface emission process, the large densities of states associated with the flat parts of, respectively, the third band in the middle of the $\Gamma N$ line and the second band near point $N$. The first structure $A$, cannot be directly assigned to a maximum of the density of states on the corresponding band scheme section and poses a problem. In spite of

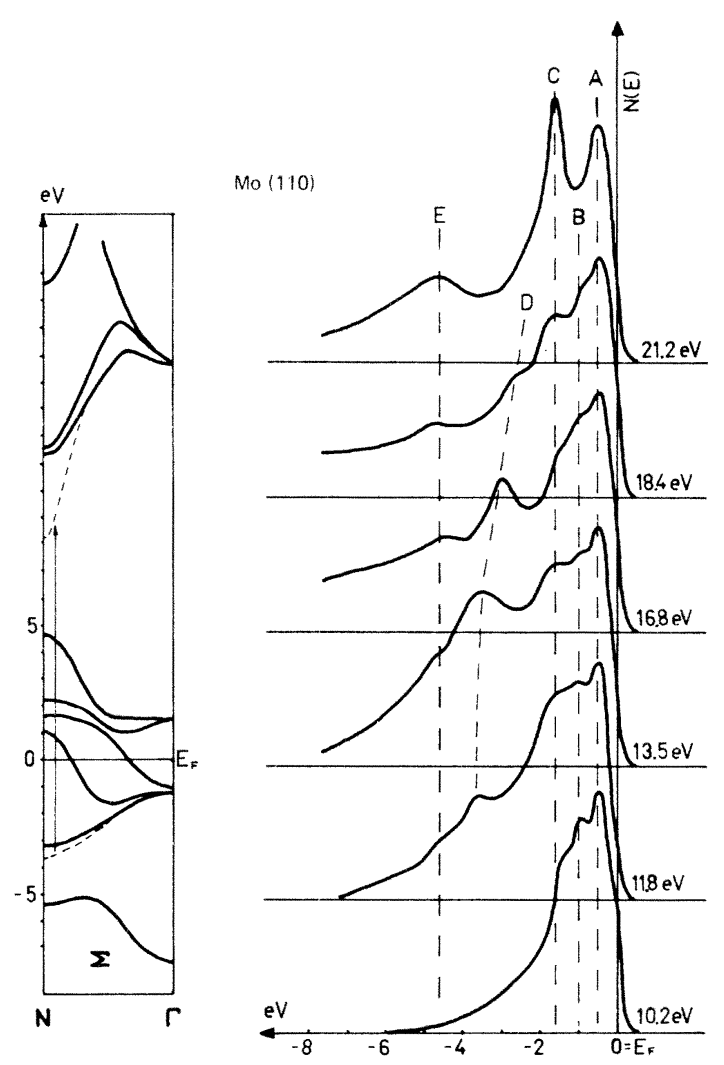

FIG. 3. Experimental spectra of photoelectrons emitted normal to the (110) surface for photon energies between 10.2 and $21.2 \mathrm{eV}$ (right-hand side). Calculated band structure of molybdenum along the $\Gamma N$ symmetry line where the observed direct transition is indicated by an arrow. 
its similarity in position $\left(0.5 \mathrm{eV}\right.$ below $\left.E_{F}\right)$ to surface-state structure observed on the (100) plane, its poor sensitivity to surface contamination points to a different origin. As an equivalent maximum of emission is observed on tungsten ${ }^{4}$ where it is assignable to surface emission from the extremum of the fourth band at point $\Gamma$, a possible explanation is that the real band structure of molybdenum is closer to that of tungsten than the calculation predicts in this region. We will evoke other possibilities in the discussion.

(c) The last stationary peak, labeled $E$ on the figure, is very interesting because it reflects a maximum of occupied states in the large gap owing to the $s-d$ hybridization in this direction of the Brillouin zone. This fact, added to its large sensitivity to surface contamination (where it disappears for a very weak exposure to residual gases while the other structures remain unaffected), gives good experimental evidence for postulating its origin in a surface-state level situated in the $s-d$ hybridization gap as predicted by Forstman, Pendry, and Heine ${ }^{19,20}$ in previous theoretical work. As for the (100) surface spectra we have not observed any emission from high-density regions of the lowest band.

\section{DISCUSSION AND CONCLUSION}

The first remark of this discussion is to underline the very strong similarity between our results and the equivalent ones obtained on tungsten. ${ }^{4}$ Spectra drawn for the same photon energies present structures of bulk origin which are mostly the same for the two materials with only a little difference in their intensities. Peaks near the Fermi level, at the center of the $d$ band, are quasi-identical in the initial energy position. Some important differences occur only for peaks situated at the bottom of the $d$ band: for example, on the (100) face, structure $D$ corresponding to the maximum of the first band between points $\Gamma$ and $H$ is found at $-4.5 \mathrm{eV}$ below $E_{F}$ for tungsten and at $-3.5 \mathrm{eV}$ for molybdenum. This important experimental difference reflects the $d$-bandwidth difference of the two metals predicted by the RAPW calculations. The surface resonant peak on the (100) face is present on both metals; inversely, structure attributed to a surface state on (110) molybdenum was not observed on tungsten. The explanation of this fact remains obscure but it could be due either to a slight accidental contamination of the $W$ surface or to an effect of physical surface conditions differences in the two experiments (roughness, for example). This latter hypothesis is suggested by the absence (contrary to the $\mathrm{W}$ case) in our high-energy spectra of a large sec- ondary emission which is not entirely attributable to the relatively poor transmission of our analyzer system in the low-energy range.

A second point worth discussing is the "corrections" drawn on the calculated band scheme to give a better fit between expected and observed structures. Several reasons can be proposed to justify these corrections: relative inaccuracy of the band-structure calculation, deformation of the real band structure near the surface owing to the presence of the surface itself, or some effect of our finite angular resolution which may include states other than those contained in the one-dimensional calculated curve. It has not been possible from the experiment to make an unambigous choice among these hypotheses. It may be noted, however, that two of the larger misfits [the shift toward higher values of the maximum of the first band for the (100) face and the severe reduction of the large gap above $E_{F}$ for the (110) face] were also observed on tungsten where they were attributed, respectively, to a narrowing of the $d$ band

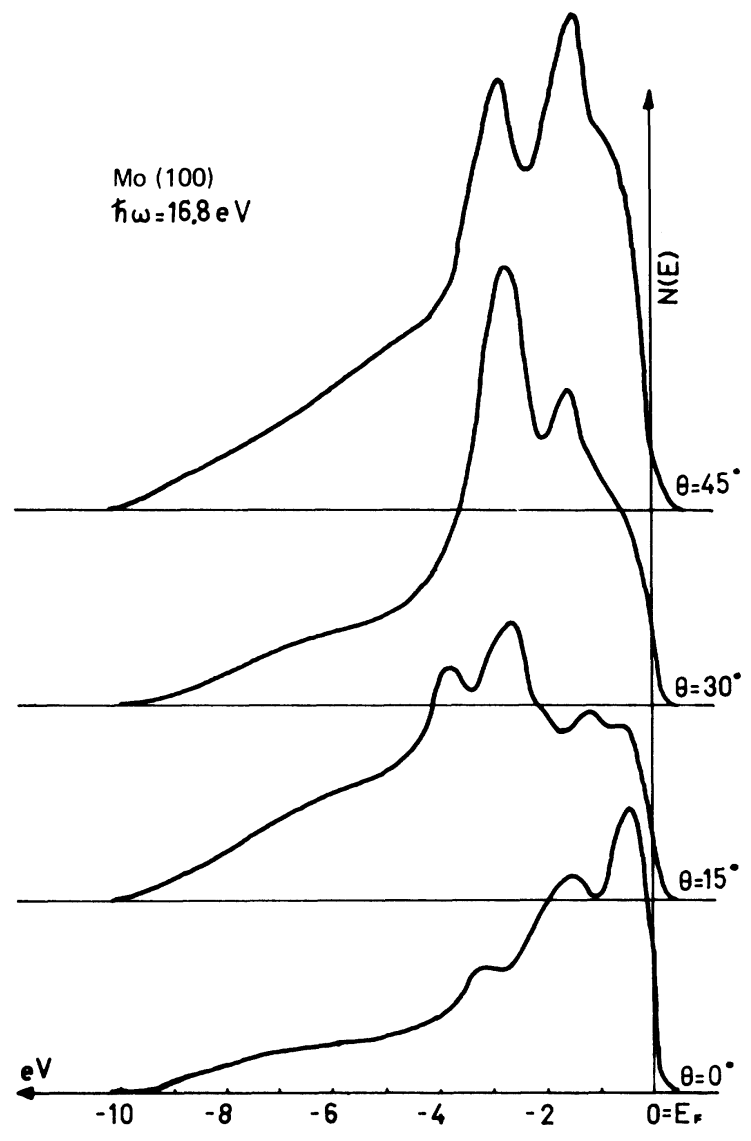

FIG. 4. Experimental spectra measured on the (100) face, $\hbar \omega=16.8 \mathrm{eV} . \theta$ is the polar angle between the surface normal and the axis of the analysis solid angle. Azimuthal angle is not determined. 
near the surface and to an angular resolution effect. In terms of band deformation near the surface, the large discrepancy found between the expected and observed position of peak $A[(110)$ face, Fig. 3] could be interpreted by a splitting of the nearly degenerate parts of bands $2-4$ at the symetry point $\Gamma$, by the proximity of the surface as a perturbation adding its effect to the spin-orbit one.

The last point concerns the validity of the hypothesis used to interpret our experimental results. Essentially we suppose the conventional direct interband transition emission model, the $K_{11}$-conservation approximation for excited electrons escaping through the surface, and the assumption of a possible surface emission process where electrons are excited directly from surface atoms to plane-wave states in the vacuum. Existence of direct transition emission is well demonstrated by characteristic peaks related unambiguously to the band structure. The $K_{11}$ conservation approximation is at first intuitively supported by the large angular effect observed when the analysis direction for emitted electrons varies (as is shown in Fig. 4 where $\hbar \omega$ is equal to $16.8 \mathrm{eV}$ and $\theta$ is the angle between the analysis cone axis and the surface normal), the azimuthal direction being undetermined. It is also justified by the appearance of only the direct transition emissions relative to the corresponding directions of the Brillouin zone on the two faces studied. The surface emission concept is used here to take accounit of the structures reflecting initial-state densities although such structures can be also interpreted in terms of a nondirect transition process. This requires some justification. The first, already discussed above, is the similarity of our results with those of tungsten $^{4}$ obtained in the same experimental conditions and where good experimental evidence was found to eliminate nondirect transition effects. The second is the fact that in our results regions of high densities of initial states in other parts of the Brillouin zone (near the symmetry point $P$, for example) do not give any contribution in measured spectra along the explored directions. Finally, the third is in the directional effect (Fig. 4) which shows all the structures varying in initial energy with collection angle in disagreement with the nondirect transition emission characteristics.

In conclusion: (i) Results have been presented on the electronic structure of single-crystal molybdenum surfaces by directional photoemission spectroscopy. (ii) All structures observed have been coherently connected to the calculated band structure within a model assuming two types of contribution, bulk three-step emission (direct transitions) and surface emission processes. (iii) Good experimental arguments have been found to confirm the surface resonant level theoretically predicted just below $E_{F}$ on the (100) face and to assign the emission from $-4.5 \mathrm{eV}$ below $E_{F}$ on the (110) face to surface states situated in the $s-d$ hybridization gap and theoretically predicted by Forstman, Pendry, and Heine.

This work was supported by the Comite d'Actions Thematiques Programmées “Surfaces” contract No. A655-1704.
${ }^{1}$ For a review see N. V. Smith, Crit. Rev. Solid State Sci. 2 , 45 (1971).

${ }^{2}$ N. E. Christensen, Phys. Status Solidi B 52, 241 (1972).

${ }^{3}$ N. V. Smith, Phys. Rev. B 3 , 1862 (1971).

${ }^{4}$ N. E. Christensen and B. Feuerbacher, Phys. Rev. B 10, 2349 (1974); 10, 2373 (1974).

${ }^{5}$ C. N. Berglund and W. E. Spicer, Phys. Rev. 136, A1030 (1964); 136, A1044 (1964).

${ }^{6}$ G. D. Mahan, Phys. Rev. B 2, 4334 (1970).

${ }^{7} \mathrm{~N}$. W. Ashcroft and W. L. Schaich, in Proceedings of the Electronic Density of States Symposium, edited by L. H. Bennett, Natl. Bur. Stds. (U.S. GPO, Washington, D. C., 1971).

${ }^{8}$ C. Caroli and D. Lederer-Rozenblatt, Phys. Rev. B $\underline{8}$, 4552 (1973).

${ }^{9}$ P. J. Feibelman and D. E. Eastman, Phys. Rev. B $\underline{10}$, 4932 (1974).

${ }^{10}$ E. Al Khoury Nemeh, R. C. Cinti, and J. B. Hudson, J. Phys. (Paris) Lett. $\underline{35}$, L179 (1974).
${ }^{11}$ S. J. Atkinson, C. R. Brundle, and M. W. Roberts, Chem. Phys. Lett. 24, 175 (1974).

${ }^{12}$ K. A. Kress and G. J. Lapeyre, J. Opt. Soc. Am. $\underline{60}$, 1681 (1970).

${ }^{13}$ Creusot-Loire Instrumentation, $15 \mathrm{Av}$. J. Jaurès, 94201 Ivry-sur-Seine.

${ }^{14}$ B. Feuerbacher and B. Fitton, Phys. Rev. Lett. $\underline{30}$, 923 (1973).

${ }^{15}$ B. J. Waclawski and E. W. Plummer, Phys. Rev. Lett. 29, 783 (1972).

${ }^{16} \mathrm{E}$. W. Plummer and J. W. Gadzuk, Phys. Rev. Lett. 25, $1493(1970)$.

${ }^{17} \mathrm{M}$. C. Desjonquères and F. Cyrot-Lackman (unpublished).

${ }^{18}$ R. Feder and K. Sturm, Phys. Rev. B 12, 537 (1975).

${ }^{19} \mathrm{~F}$. Forstman and V. Heine, Phys. Rev. Lett. 24, 1419 (1970).

${ }^{20}$ F. Forstman and J. B. Pendry, Z. Phys. (Leipz.) $\underline{235}$, 75 (1970). 